\title{
Detection of Noncircularity and Eccentricity of a Rolling Winder by Artificial Vision
}

\author{
Christophe Doignon \\ Laboratoire des Sciences de l'Image, de l'Informatique et de la Télédétection, UMR CNRS 7005, Équipe de Recherche \\ Technologique en Enroulement-ERT $n^{\circ}$ 8, Boulevard Sébastien Brant, 67400 Illkirch, France \\ Email: christophe.doignon@ensps.u-strasbg.fr

\section{Dominique Knittel} \\ Laboratoire des Sciences de l'Image, de l'Informatique et de la Télédétection, UMR CNRS 7005, Équipe de Recherche \\ Technologique en Enroulement-ERT $n^{\circ}$ 8, Boulevard Sébastien Brant, 67400 Illkirch, France \\ Email:dominique.knittel@ipst-ulp.u-strasbg.fr
}

Received 31 July 2001 and in revised form 13 March 2002

\begin{abstract}
A common objective in the web transport industry is to increase the velocity as much as possible. Some disturbances drastically limit this velocity. Time-varying eccentricity of the rolling winder is one of the major disturbances which affect the quality of the rolling winder. This unsuitable factor can lead to a web break for a high-speed winding process. The main contribution of this work is to offer a new measurement technique that is able to provide on-line the estimation of the roll radius and its variations with a subpixel accuracy. A key feature within this work is the contour curvature classification by means of wavelets decomposition of the edge orientation function. We also propose a new model accounting for the increasing radius of the rolling winder, which confirms the experimental results and the reliability of the proposed approach.
\end{abstract}

Keywords and phrases: shape-preserved filtering, curvature analysis, wavelets decomposition, contours classification, ellipse fitting, pose from ellipse.

\section{INTRODUCTION}

Products made with paper, textile, metal, or polymers need to be winded and unwinded during an industrial process. A common objective is to increase as much as possible the web transport velocity while controlling tension of the web. However, there exist many sources of disturbances like noncircularity of the roll, eccentricity of the roll, web sliding, temperature variations, variations in motor torque, $\ldots$ which are some limiting factors of performances. Since there exists a coupling introduced by elastic property of the web, disturbances are transmitted to the web tension, resulting in a web break or fold. In an attempt to reduce these harmful effects, recent works on modelization and control for web handling applications are promising and can be found, for example, in $[1,2,3]$. They are mainly based on PID, $H_{\infty}$ robust control, fuzzy logic, or neural network approaches [4]: due to a widerange variation of the roll radius, system dynamics considerably change (inertia is proportional to the fourth power of the roll radius). Few works in the image processing or computer vision fields applied to winding systems have been yet published. However, the use of a visual sensor through digital image analysis for supervising the quality of the roll is being emerging $[5,6]$. Figure 2 shows two reels with equal length but winded under different conditions: the speed and tension of the web were constant for the left reel while a too strong and variant tension and speed magnitude were applied on the web for the right reel.

This paper is built on some relevant image segmentation features dedicated to the roll radius estimation and its variations. It is organized as follows. In Section 2, we present the methodology of the contour-based image segmentation: fast edges detection, gradient phase smoothing, contours classification, and outer edge contours extraction of the roll. For this application of visual motion in presence of noisy data, we focus on noise filtering for edge orientation analysis. Section 3 is dedicated to an elliptic curve fitting based on the Cholesky decomposition since some elliptic arcs have been selected to approximate the roll shape. In order to validate the mean value of the estimated roll radius, a simple model accounting for the roll radius increase and based on pure kinematic considerations is proposed. The last section is devoted to a discussion about the results obtained with the implemented technique on the experimental setup. Particularly, we compare the roll radius estimations provided by the proposed method and a series of measurements on static rolls with a mechanical device. 


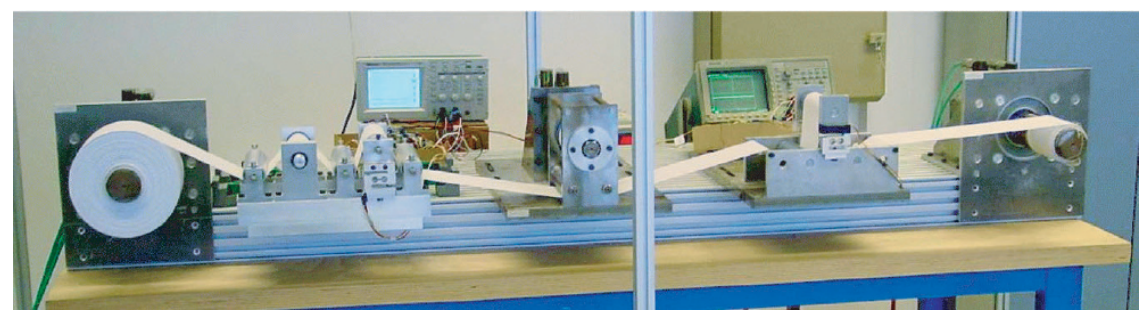

FIGURE 1: The experimental winding equipment.

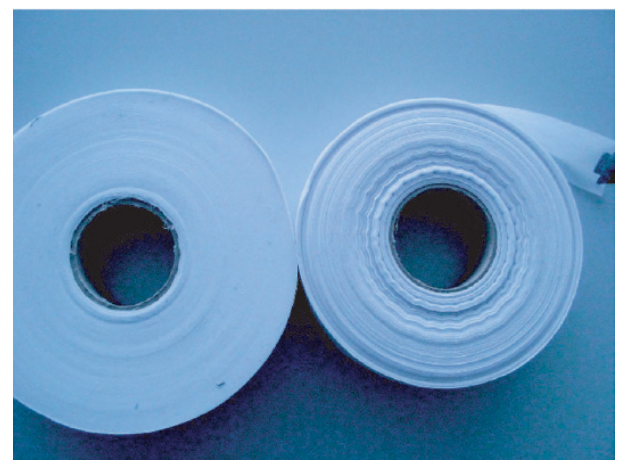

FIGURE 2: High quality (left) and low quality (right) of the resulted reel.

\section{IMAGES SEQUENCE ANALYSIS}

A fundamental requirement in vision systems is the ability to extract from digital images primitives relevant to the observed scene. Edge contour segmentation and curve parametrization are therefore important stages for representing boundary information in a structural form. Since we deal with grey level images, some classical preprocessings like contrasts enhancement by histogram equalization and nonlinear median filtering (suppression of peak noise) are performed but are not described here.

\subsection{Contours detection}

Since we are concerned by the analysis of visual motion in a time-varying image sequence, we are interested in selecting pixels that significantly contribute to the motion field. The partial temporal derivative ( $p t d$-image) is computed on two consecutive frames (see Figure 4) in order to build a fast edge detector as follows:

- in an attempt to detect "pixels in motion," the absolute value of image subtraction is computed and applied to consecutive frames (the ptd-image),

- locations of pixels (for which absolute difference in the ptd-image are greater than a threshold) are stored in a list. Detection of edges is performed with a directional filter in the original grey level image only for pixels having corresponding location in the list and also for its eight-connected neighboring pixels. We choose the Canny filter as a directional gradient filter since it provides the edge orientation. To make the implementation as efficient as possible, we use the separability property of the Canny filter (this filter is often approximated with the first derivative of a continuous Gaussian function $g(x, y)$ ), discrete kernels components are scaled and approximated by integers and lookup tables are also used to store the gradient magnitude entries. Furthermore, we fixed the standard deviation parameter according to Canny's criteria (good detection, good localization, and unicity of response) in order to consider always a unique set of masks. It is clear that this is a frequency limitation since this fix the width of greylevel transitions. With a standard deviation value of 1.5 for the Canny filter parameter, we select the fifteen most significant components of the two discrete convolution kernels as an approximation of the continuous convolution kernels for the two operators $\partial g / \partial x$ and $\partial g / \partial y$ (see Figure 5). This yields two $(3 \times 5$ and $5 \times 3$ ) masks. By this way, consuming time (the computational time is about 18 minutes (see Section 4 for details about the platform)) is significantly reduced and static background of the observed scene is removed (see Figure 6).

Canny's detector always yields edges with many pixels of thickness. Two thinning operations are performed on the extracted set of edges based on the comparison of gradient magnitude and orientation of each pixel with their neighbors within the edge (nonmaxima suppression) producing a 1-pixel wide edge: this is a requirement for the apply of the contour following method [7] to edges tracking. Edges tracking is the process of associating nearby edge points so as to create a connected boundary. At any detected edge, this process selects, among the set of the 8-nearest neighbors, the next one to include in a list (and labeled or simply deleted in the gradient image) producing a displacement through this list. This process is significantly optimized if thinning operations are previously done (this confers the ability to begin the search for the next edge with the direction of the previous displacement).

Contours in the list are merged by using a process called mutual favorite pairing: as a quality measure, a cost function can be computed for every connected path between two endpoints of distinct contours. It uses orientations and distances between endpoints of each pair of contours [8]. This process allows to merge neighboring contour chains 

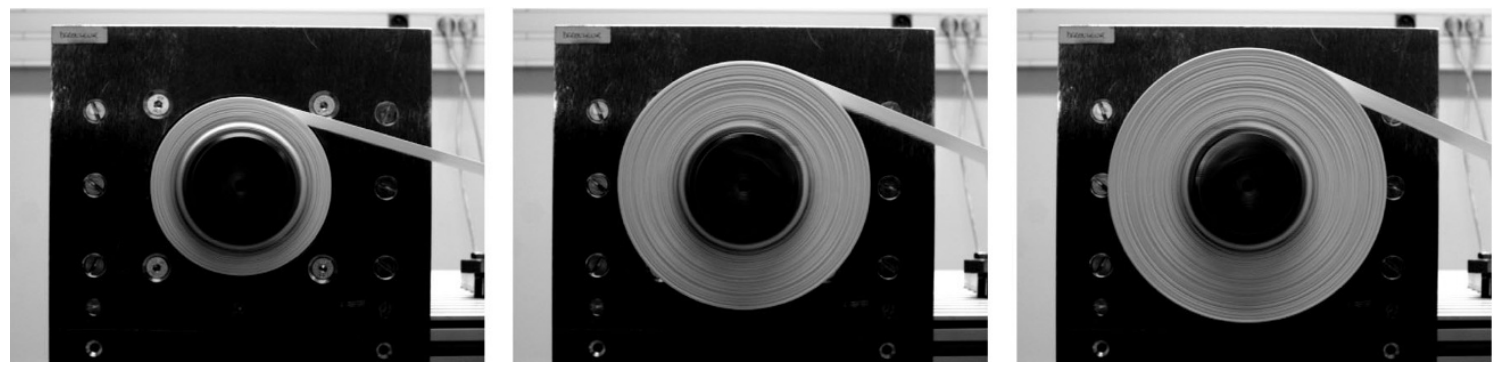

FIGURE 3: Some images of the rolling winder during the sequence.
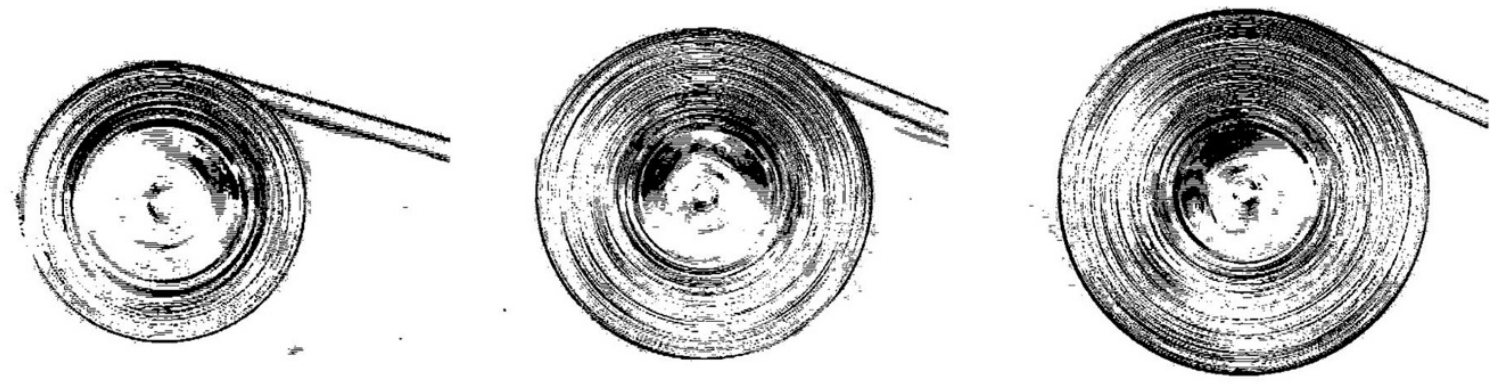

FIGURE 4: Some substractions of two successive frames: irrelevant static background has been removed from these three ptd-images (a binarization has been carried out for a good print).

into a single contour. Contours that are too short for a future classification are rejected.

\subsection{Gradient phase smoothing}

The sequence of operations of most computer vision systems begins by detecting and locating some features in the input images. To achieve this, edge contours have to be expressed in a form suitable for classification. A global representation based on set of moments or Fourier descriptors is unsuitable when partial objects or image features are involved, and it is not suited for object location. Local representation as edge orientation function (extracted from the gradient phase signal), and its derivatives, is more interesting since it is not strongly affected by partial occlusions and it allows to define differential invariants under scaling, translation, and rotation transformations. Furthermore, the edge detection gives this information in a nearly continuous space as Canny filter contributes to gradient phase smoothing. In this representation, local edge orientation is expressed as a one-dimensional function of $\theta(s)$ where $s$ is the curvilinear abscissa. Discontinuities in $\theta(s)$ and its derivatives mark limits between elementary fragments of a contour and reflect transitions between them.

A major problem of methods relying on the orientation function and its derivatives is their sensitivity to noise. However, application of classical filtering has harmful effects like attenuation of discontinuities, change of shape, blurring effects, and so forth. This complicates the detection of singular points. Consequences are more severe for smoothed portions of contours for which discontinuities related to singular points only appear in the high-degree derivatives of the orientation function. A simple noise filtering procedure may lead to a delocalization, or even to a loss of these salient points. Wuesher and Boyer [9] smooth at first the curvature function (first derivative of the orientation) using a Gaussian kernel with a low smoothing parameter in order to reduce the blurring effect and to preserve valid high frequency shape information. However, in that way, the noise was not well attenuated and they were constrained to apply a nonlinear filter to reduce the remaining noise. The polygonal approximation of curves proposed by Pikaz and Dinstein [10] significantly reduces the noise but the implemented algorithm is complex and highly time-consuming. Our objective is to find a linear smoothing filter that is a trade-off between a maximum reduction of noise and a good preservation of high frequency informations in the orientation (or gradient phase) function.

Weiss [11] defined what he called the power preserving filter as follows: considering the Taylor expansion $f(x)=$ $\sum\left(f^{(n)} / n !\right) x^{n}$ of a function $f(x)$ representing the input signal, a filter of order $l$ is a filter which preserves the power $x^{n}$ up to order $l$ with respect to the convolution $F_{l}(x, \sigma) * x^{n}=$ $x^{n}$, (for $\left.n=0, \ldots, l\right)$ where $F_{l}(x, \sigma)$ is the impulsional response of the filter. Weiss showed that moments of such filters, defined by

$$
m_{n}=\int_{-w}^{w} x^{n} F_{l}(x, \sigma) d x,
$$

vanish up to the order $l$ except for the 0th moment which is equal to 1 . From this, a Gaussian filter is of order $1(g(x, \sigma) *$ 


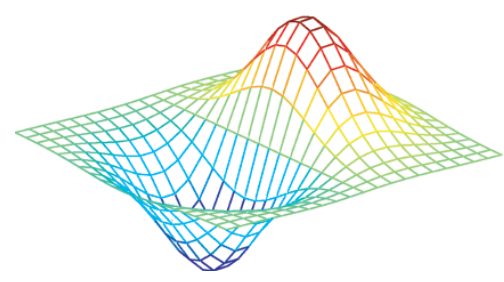

(a)

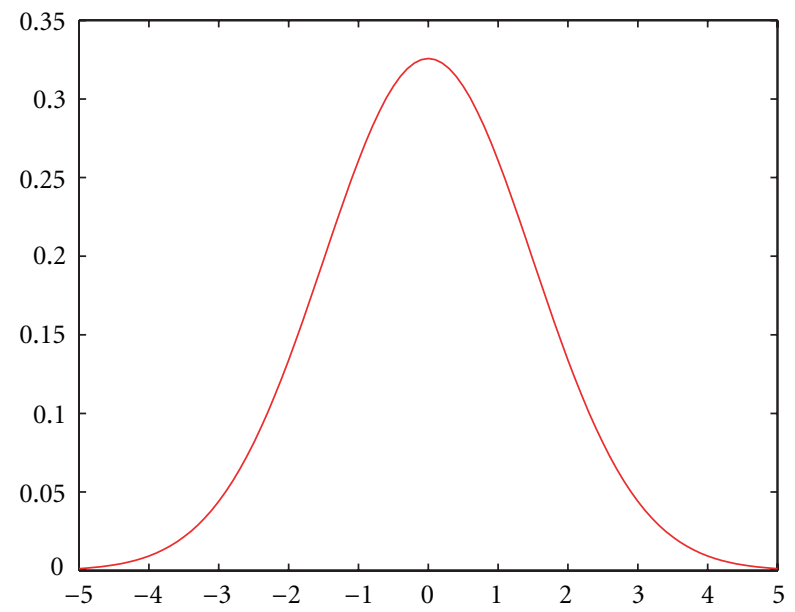

(c)

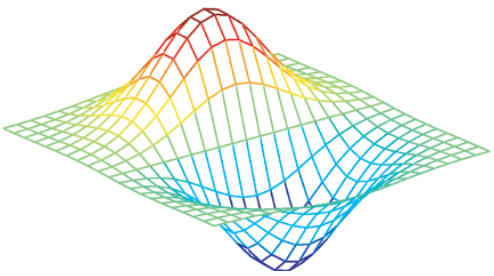

(b)

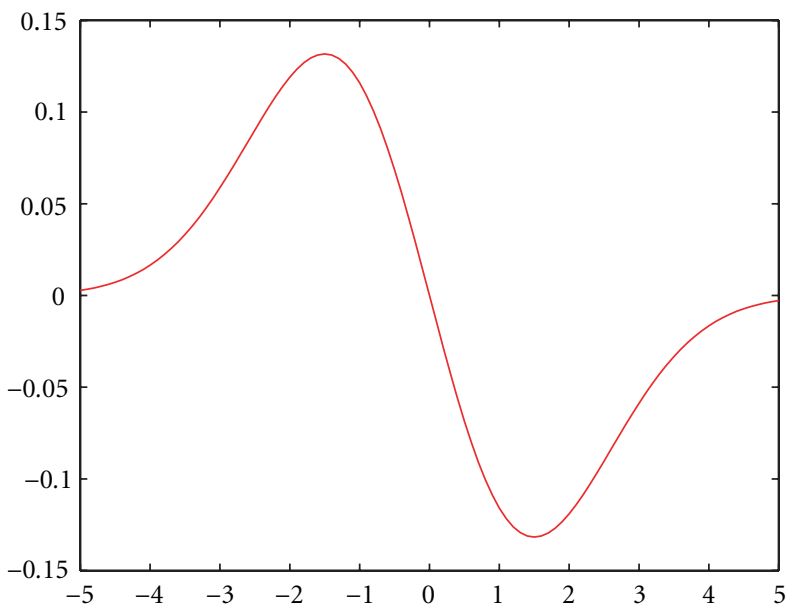

(d)

Figure 5: The first derivative of a Gaussian as an approximate solution of Canny's criteria for edges detection $(\sigma=1.5)$. (a) and (b) partial derivatives of the 2D Gaussian filter with respect to $x$ and $y$, (c) the 1D Gaussian function, and (d) its first derivative.

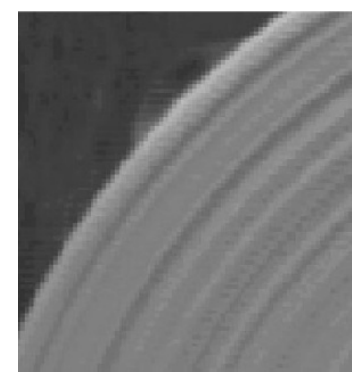

(a)

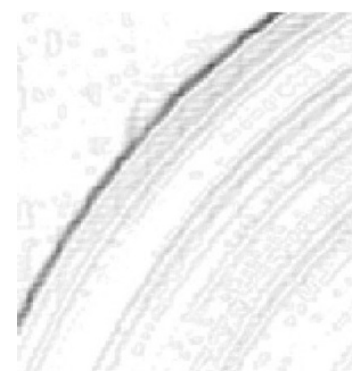

(b)

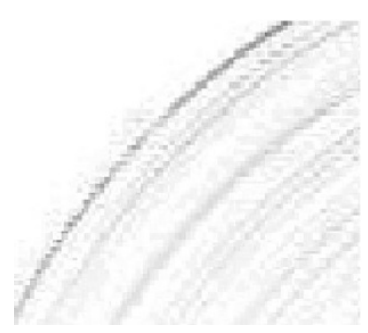

(c)

Figure 6: (a) Part of original image, (b) partial temporal derivative on consecutive frames, (c) gradient magnitude computed in the original image for motion pixels and their neighborhood.

$x=x$ ). Errors due to additive terms and causing blurring effect or bias are expressed by

$$
\mathscr{C}_{\text {Weiss }}=\frac{\left\|m_{l+1}\right\|}{(l+1) !} \frac{\sigma^{l+1}}{s_{0}} .
$$

The smaller (2), the best is the filter. $\sigma$ is the smoothing parameter, $l$ is the order of the filter, and $s_{0}$ is a scale of the smoothed function. In our case, it can be taken as the length of the shortest contour. To have a low phase distortion, the phase function of linear lowpass filters should be quasi-linear in the bandpass. Among classical linear filters, we choose the Butterworth, Chebyshev and elliptic filters for their frequency characteristics (see Figure 7). Corresponding filters will then be first order filters preserving the shape of contours approximated with quadratic functions of coordinates like ellipses (see Figure 8). It is clearly not the case with the Gaussian filter $\left(g(x, \sigma) * x^{2}=x^{2}+\sigma^{2}\right)$. 


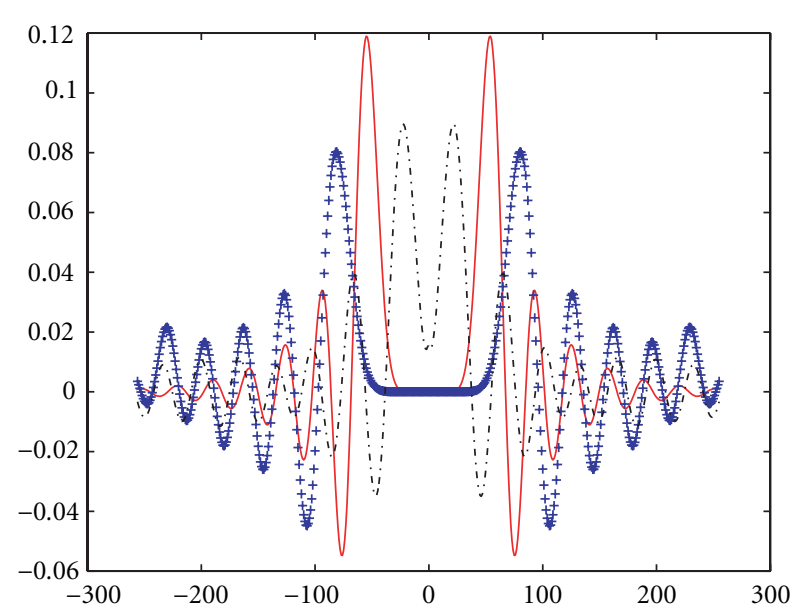

(a)

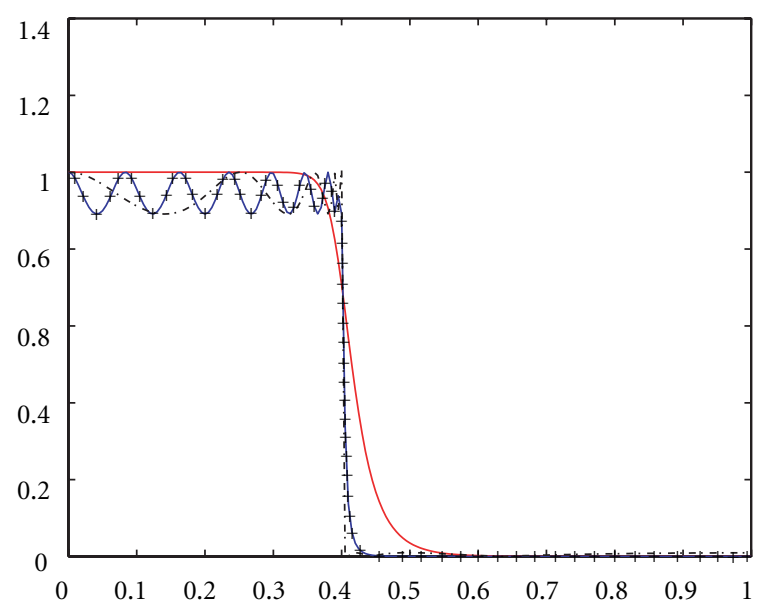

(b)

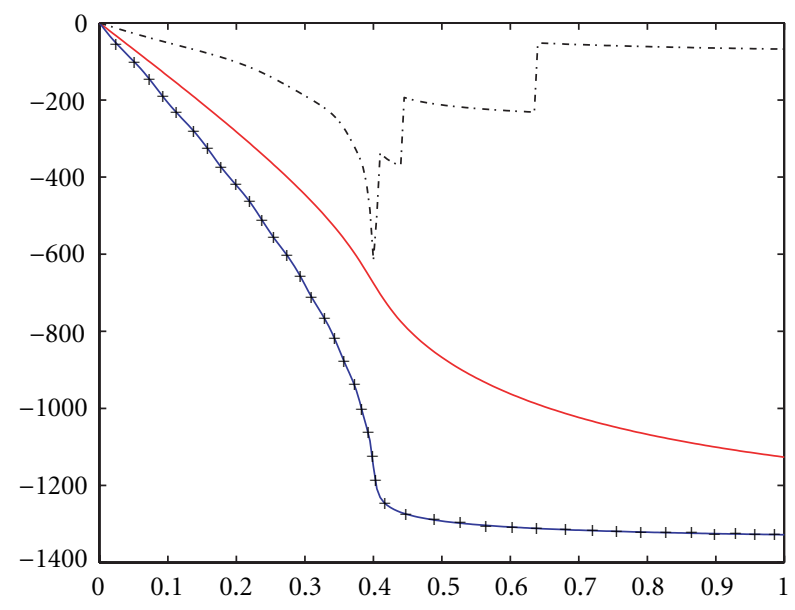

(c)

FIgURE 7: (a) Impulsional responses, (b) magnitude-frequency responses, (c) phase-frequency responses for Butterworth (solid), Chebyshev (+), and elliptic (dashed) filters.

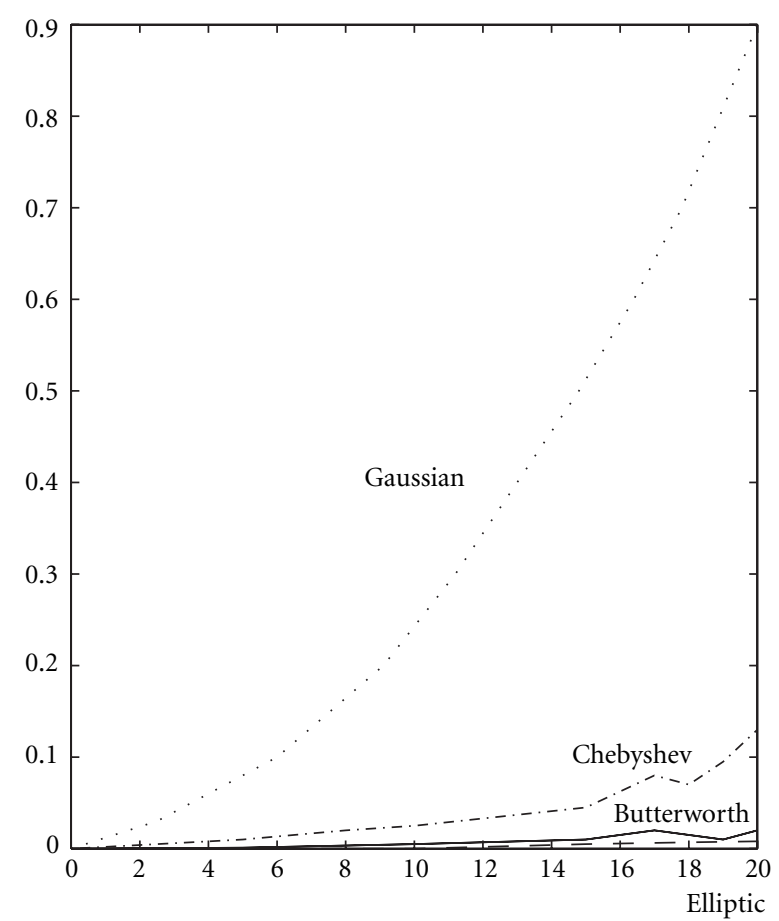

Figure 8: The preserving shape criterion $\mathscr{C}_{\text {weiss }}$ with respect to the smoothing $\sigma$ parameter: Chebyshev (-..), elliptic filter (-), Butterworth filter (solid), Gaussian filter (....).

\subsection{Classification}

\subsubsection{Multiscale detection of gradient phase discontinuities}

Classification of contours is an ubiquitous problem in image segmentation. For high-level programs, it is convenient to characterize a contour with some intrinsic few descriptors rather than a set of many pixels. Since inflexion points and also inflexion lines (tangent lines at inflexion points) are invariant under projective transformations, they are good candidates for such descriptors $[12,13]$. Consequently, the main objective is to detect curvature discontinuities along a contour. It is essential to conceive an algorithm for curvature discontinuities detection which provides the same location of inflexion points for any viewpoint. This is of most importance for image contours matching methods based on differential invariants $[12,13]$ or corners [14] for instance. The orientation function analysis proposed in this paper is based on a multiscale scheme [15]. It used the wavelet transform which is adequate for detecting discontinuities in nonstationary signals with finite energy. In image processing, wavelets have been used in image compression, contours/features detection, and threshold selection of grey level images for instance. A wavelet is a function with null average and it is well localized in both time and frequency spaces. The prototype wavelet $\psi_{a}(x)$ (one basic function of the decomposition) can be thought of as a bandpass filter. The more interesting property for our application is that the wavelet does not reflect regularity of the function but rather of its 


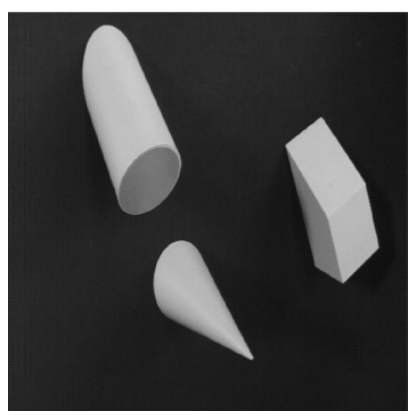

(a)

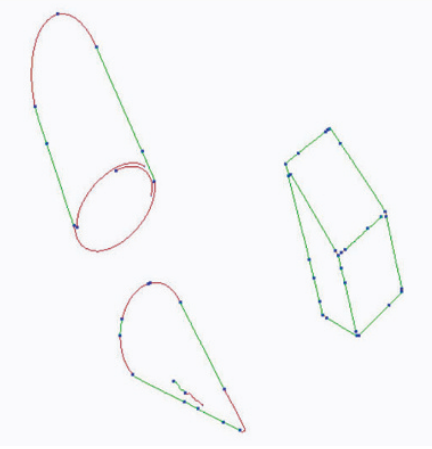

(b)

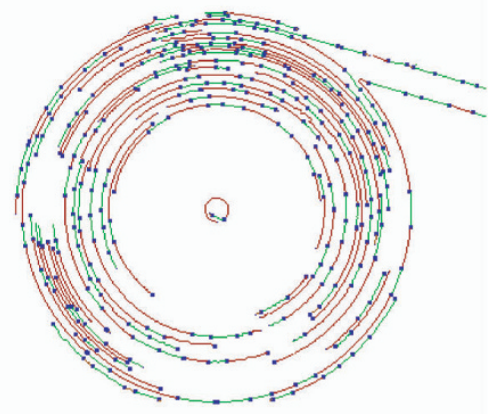

(c)

FIgURE 9: Curvature discontinuities detection. Fragment is defined between two discontinuities inside a contour. (a) a test image, (b) curvature discontinuities detection, (c) curvature discontinuities found for the roll.

derivatives $([16,17])$. For a given scale, it takes a small value for the regular part of the signal and it explode at points where the signal or its derivatives undergo brutal changes. Then, wavelet transform can disclose both the discontinuities of the orientation function $\theta(s)$ and those of its first derivative (local curvature), second derivative (variations of the local curvature), and so forth. Considering the secondderivative of a Gaussian function (the Marr's wavelet) as the prototype wavelet, this function vanishes quickly in both time and frequency spaces. Zero-crossings of the wavelet transform belong to a set of lines which converge at inflexion points of the $\theta(s)$ function for different scales $a$. The choice of a continuous wavelet is more convenient than a dyadic wavelet for the study of the wavelet modulus along these lines [14].

The wavelet transform $W_{f}$ is defined as

$$
W_{f}(a)=\left\langle f, \psi_{a}(x)\right\rangle=\frac{1}{\sqrt{a}}\left\langle f, \psi\left(\frac{x}{a}\right)\right\rangle=\int_{-\infty}^{\infty} f(x) \psi_{a}(x) d x
$$

with

$$
\psi(x)=\frac{2}{\sqrt{3 \sqrt{\pi}}}\left(1-x^{2}\right) e^{-x^{2} / 2} \quad \text { as a prototype wavelet. }
$$

Discontinuities detection gives as output a set of points which defines the ends of simple fragments composing a contour (see Figure 9). Contour fragments are bounded by curvature discontinuities or by an endpoint and a discontinuity. In other words, there are as many contour fragments as curvature discontinuities detected minus one all along a contour. The wavelet transform modulus of these points is above a threshold. To improve their localization, we check neighborhoods afterwards. The aim of thresholding is to eliminate false discontinuities due to remaining noise in the orientation function after the linear smoothing operation. We have to estimate statistics of noise in the wavelet transform of the smoothed orientation function. We model the whole process by a succession of lowpass filters followed by the bandpass filter corresponding to the wavelet transform. The variance $\sigma_{m}^{2}$ of the output noise can be computed if the input is the orientation function $\theta(s)$ to which white noise $n\left(0, \sigma_{n}^{2}\right)$ is added [15]. We choose a threshold value greater than $\sigma_{m}$. In our experiments, a threshold value of $2.5 \sigma_{m}$ has proven to be sufficient. Thus, we classified image contours between two discontinuities (or between an endpoint and a discontinuity) as follows: the wavelet transform of an orientation function corresponding to a straight line fluctuates around zero, on the contrary it keeps a constant sign for a curve without inflexion point (like an elliptic arc for instance). Therefore, by counting the zero-crossings of the wavelet transform of orientation function we can know whether it corresponds to a straight line or not (see Figures 10 and 11).

\subsubsection{Location of the outer contour}

A list of contour fragments is built with fragments not corresponding to straight line. Some small fragments are rejected because they can be interpreted as the projections of limits between inner layers of the roll and an approximation of such portions of contours with a simple ellipse fitting is untractable (as a limit, these arcs look like a noisy line). However, since all contour fragments contribute to the same center (but not the same radius), we derive an estimate for the localization of this center based on a modified circular fitting. To find that global center $C=\left(x_{c}, y_{c}\right)^{T}$, we can proceed in three stages through the list of contour fragments:

(1) For any pair of pixels $Q_{i}=\left(x_{i}, y_{i}\right)^{T}$ and $Q_{j}=\left(x_{j}, y_{j}\right)^{T}$ of the same contour fragment $c$, its contribution to a circular fitting is computed as

$$
\begin{aligned}
& \left(x_{i}-x_{c}\right)^{2}+\left(y_{i}-y_{c}\right)^{2}+r_{c}^{2}=0, \\
& \left(x_{j}-x_{c}\right)^{2}+\left(y_{j}-y_{c}\right)^{2}+r_{c}^{2}=0 .
\end{aligned}
$$

A simple difference on previous equations eliminates the radius parameter and provides a linear relation with respect to the center coordinates as unknown:

$$
\underbrace{2\left(\left(x_{i}-x_{j}\right)\left(y_{i}-y_{j}\right)\right)}_{\underline{h}_{n}^{T}}\left(\begin{array}{l}
x_{c} \\
y_{c}
\end{array}\right)=\underbrace{\left(x_{i}^{2}-x_{j}^{2}+y_{i}^{2}-y_{j}^{2}\right)}_{k_{n}} \text {. }
$$




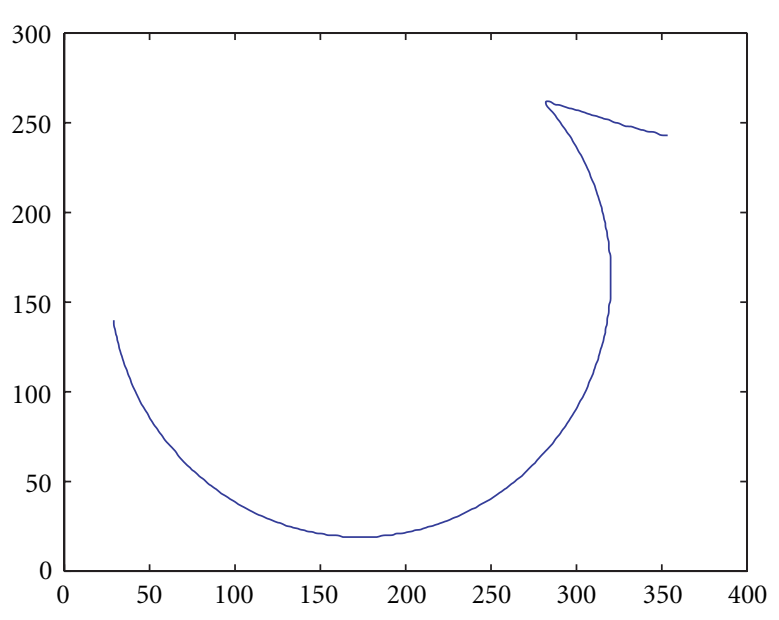

(a)

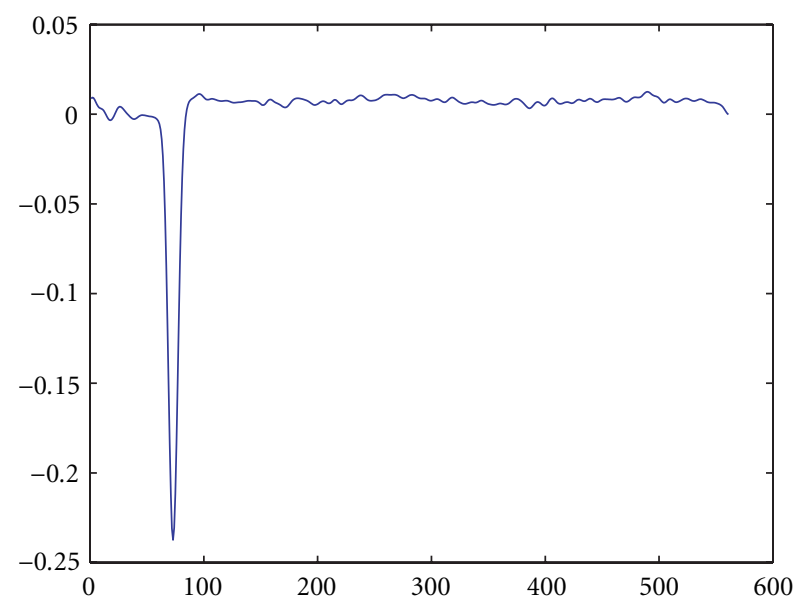

(b)

Figure 10: (a) A contour of the roll, (b) the 1D corresponding curvature variations.

For a contour fragment with $N_{c}$ pixels, $N_{c}-1$ relations as above contribute for estimating the global center.

(2) Consider the next fragment $(c+1)$ in the list of contour fragment and repeat the first stage until the end of the list is reached.

(3) Build a $\left(N_{t}\left(=N_{1}+N_{2}+\cdots+N_{n}\right) \times 2\right)$ matrix $H=$ $\left(\underline{h}_{1}^{T}, \underline{h}_{2}^{T}, \ldots, \underline{h}_{N_{t}}^{T}\right)^{T}$ and a vector $\underline{k}=\left(k_{1}, k_{2}, \ldots, k_{N_{t}}\right)^{T}$ by a simple concatenation of all equations (6) obtained from all contour fragments. The Least Mean Square (LMS) solution gives an estimate $C^{\star}=\left(x_{c}^{\star}, y_{c}^{\star}\right)$ of that global center.

We point out that the right size of (6) may be very small. A data normalization is needed to avoid numerical instability of a homogeneous linear system. Furthermore, a judicious choice of the couple $\left(Q_{i}, Q_{j}\right)$ can be simply obtained by browsing the list in both senses $\left(j=N_{c}-i\right)$ or by shifting the index $\left(j=N_{c} / 2+i\right)$. Finally, fragments are sorted according to the Euclidean distance (an average along the fragment) from that center. Farthest fragments of contours have been kept and reassembled to approximate the outer profile of the roll with an ellipse fitting (see Figure 12).

\section{ELLIPSE FITTING}

Particularly, we have been interested in approximating contour fragment with elliptic features. This kind of geometric feature strongly constrains the 3D point of view. Thus, it is salient for object localization and orientation if it can be detected in a reliable manner. An ellipse can be represented with the quadratic algebraic equation

$$
\left(\begin{array}{lll}
x & y & 1
\end{array}\right) \underbrace{\left(\begin{array}{ccc}
c & \frac{b}{2} & \frac{e}{2} \\
\frac{b}{2} & a & \frac{d}{2} \\
\frac{e}{2} & \frac{d}{2} & f
\end{array}\right)}_{E}\left(\begin{array}{l}
x \\
y \\
1
\end{array}\right)=0,
$$

with the quadratic constraint $b^{2}-4 a c<0$. Ellipse parameters are components of the vector $\underline{a}=(a, b, c, d, e, f)^{T}$. There are many ellipse fitting methods available in the literature. Methods based on Hough transform [18] are not suitable for our purpose as they are computationally too expensive. Classical least square method gives acceptable results for long arcs covering a large portion of the ellipse with Gaussian noise otherwise estimation is biased. Extended Kalman filter avoids this drawback but the problem persists in the case of shallow arcs with high curvature $([15,19])$. Porril $[20]$ suggested a biascorrected Kalman filter based on the likelihood principle to overcome this problem. Since in (7) ellipse parameters have been defined up to a scale factor, all previous methods usually assume that $f=1, a^{2}+c^{2}+b^{2} / 2=1, a+c=1$, or $\|E\|=1$. It is clear that none of these constraints is adequate to segregate ellipse from other conics. Recently, Pilu [21] proposed a method based on the minimization of algebraic distance solved with the generalized eigenvalue problem. By using a prenormalization data processing (consisting in prescaling and translating coordinates $(x, y)$ ) and Cholesky decomposition, we propose to minimize the following criterion:

$$
\mathscr{C}=\left\|X_{1} \underline{a}_{1}+X_{2} \underline{a}_{2}\right\|^{2}-\lambda\left(\underline{a}_{1}^{T} C \underline{a}_{1}+1\right)
$$

with

$$
\begin{aligned}
\underline{a}_{1} & =(a, b, c)^{T}, \quad \underline{a}_{2}=(d, e, f)^{T}, \quad \underline{a}^{T}=\left(\underline{a}_{1}^{T}, \underline{a}_{2}^{T}\right), \\
X_{1} & =\left(\begin{array}{ccc}
y_{1}^{2} & x_{1} y_{1} & x_{1}^{2} \\
y_{2}^{2} & x_{2} y_{2} & x_{2}^{2} \\
\vdots & \vdots & \vdots \\
y_{n}^{2} & x_{n} y_{n} & x_{n}^{2}
\end{array}\right), \quad X_{2}=\left(\begin{array}{ccc}
y_{1} & x_{1} & 1 \\
y_{2} & x_{2} & 1 \\
\vdots & \vdots & \vdots \\
y_{n} & x_{n} & 1
\end{array}\right), \\
C & =\left(\begin{array}{ccc}
0 & 0 & -2 \\
0 & 1 & 0 \\
-2 & 0 & 0
\end{array}\right) .
\end{aligned}
$$



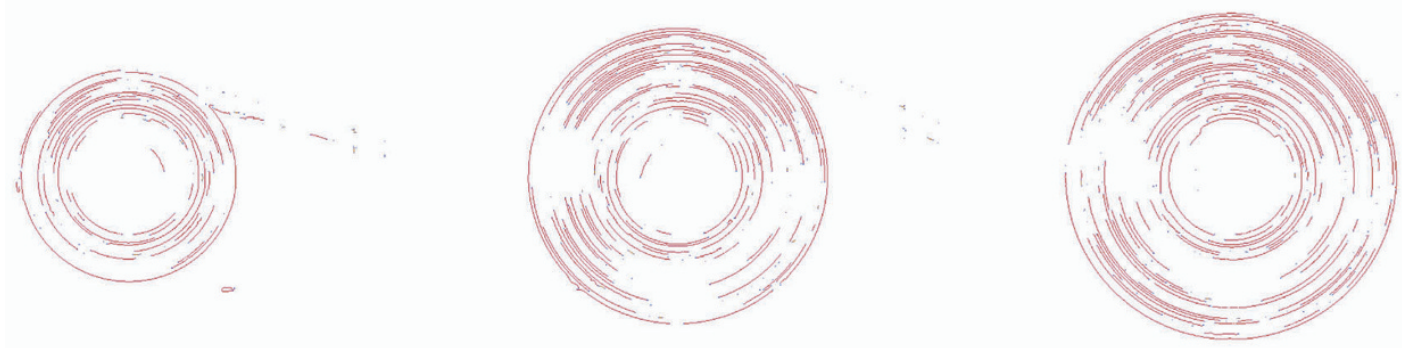

FIGURE 11: Detection of nonlinear contour fragments of the roll.
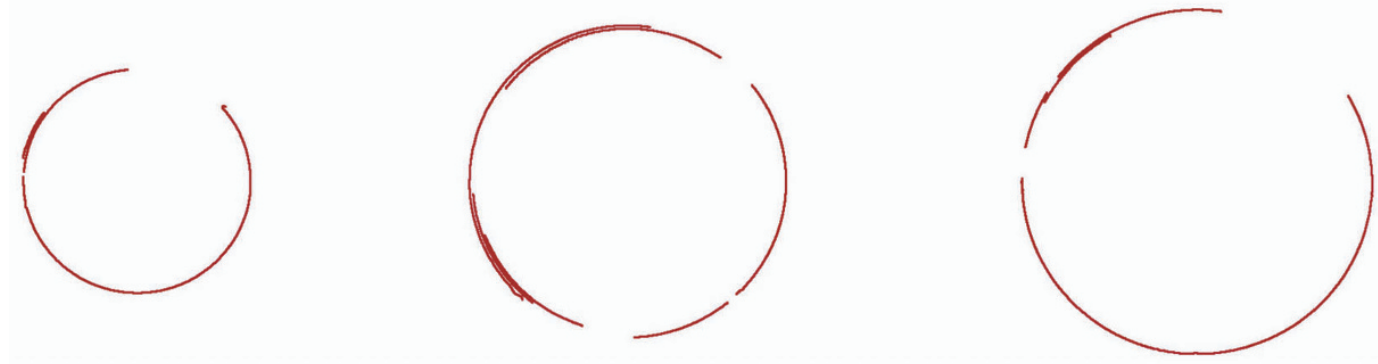

FIGURE 12: Extraction of outer contour fragments of rolling winder during the sequence.
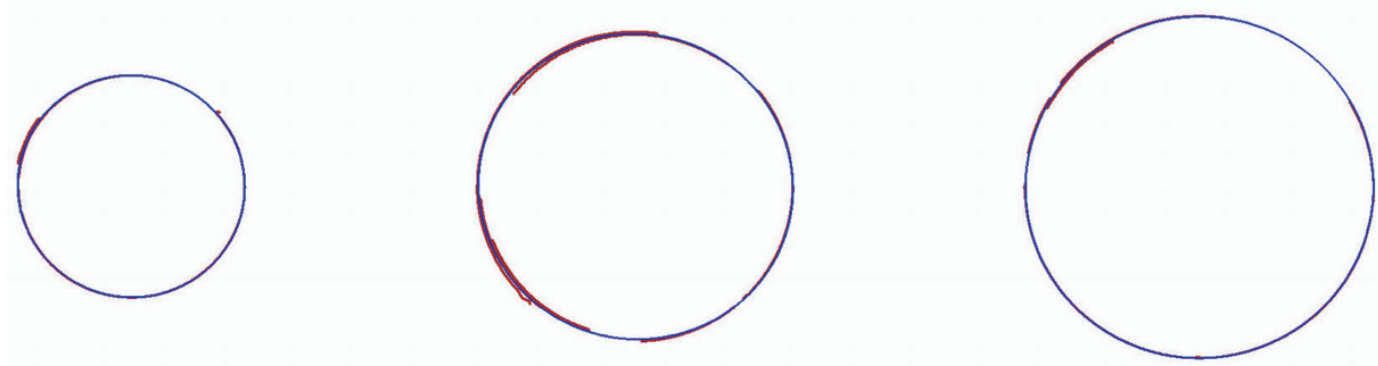

FigURE 13: Ellipse fittings of outer contour fragments of the roll.
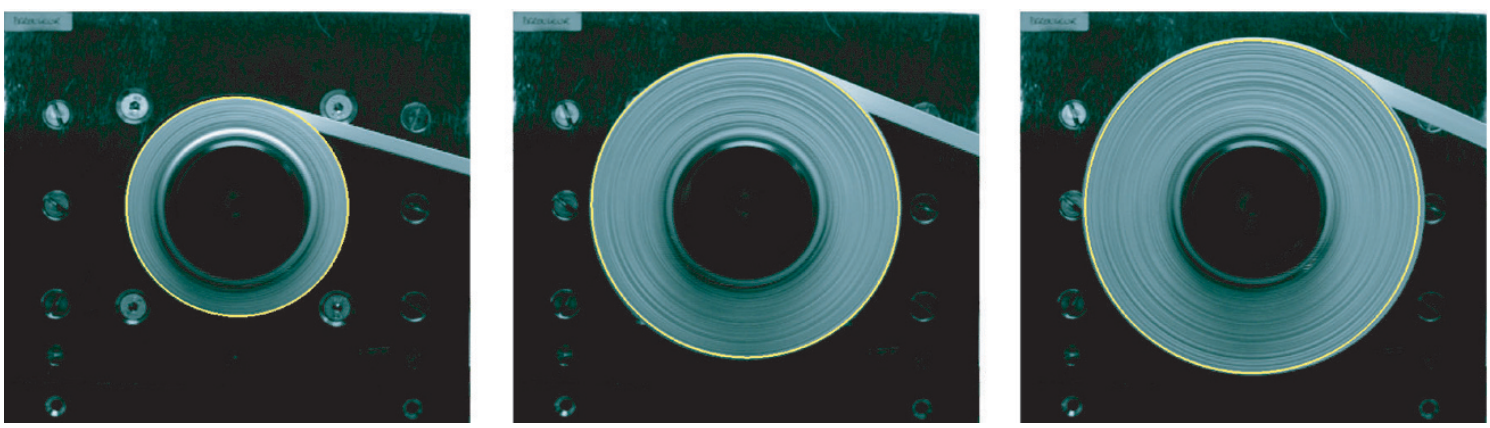

FIGURE 14: Ellipse fittings superposed to grey level images.

Derivatives of $\mathscr{b}$ with respect to vectors $\underline{a}_{1}$ and $\underline{a}_{2}$ provide an analytic solution $\underline{a}^{\star}$ for the smallest eigenvalue $\lambda^{\star}$ and with the constraint $b^{2}-4 a c=-1$, which is invariant to Euclidean transformations: 


$$
\begin{aligned}
& \frac{1}{2} \frac{\partial \mathscr{C}}{\partial \underline{a}_{1}}=X_{1}^{T} X_{1} \underline{a}_{1}+X_{1}^{T} X_{2} \underline{a}_{2}-\lambda C \underline{a}_{1}=0, \\
& \frac{1}{2} \frac{\partial \mathscr{C}}{\partial \underline{a}_{2}}=X_{2}^{T} X_{1} \underline{a}_{1}+X_{2}^{T} X_{2} \underline{a}_{2}=0 .
\end{aligned}
$$

This is the decomposition of the criterion used by Pilu but with a non-rank-deficient constraint matrix $C$. Thus, vectors $\underline{a}_{1}$ and $\underline{a}_{2}$ can be expressed by

$$
\begin{aligned}
\underline{a}_{2} & =-S_{2}^{-1} S_{12}^{T} \underline{a}_{1}, \\
S \underline{a}_{1} & =\lambda C \underline{a}_{1}
\end{aligned}
$$

with the scatter matrix $S$

$$
\begin{array}{ll}
S=S_{1}-S_{12} S_{2}^{-1} S_{12}^{T}, & S_{1}=X_{1}^{T} X_{1}, \\
S_{2}=X_{2}^{T} X_{2}, & S_{12}=X_{1}^{T} X_{2} .
\end{array}
$$

The only valid solution corresponds to the single negative eigenvalue $\lambda^{\star}$ of (12). The proposed method is a good tradeoff between computational time and unbiased results in the presence of noisy data (mainly due to quantization and thinning operations) or outliers (one can see in Figures 12 and 13 that some inner layers are also selected).

\section{POSE FROM ELLIPSE}

Here, we remember the pose recovery from an ellipse (see [22]). The problem is, given a known ellipse on the world plane and its corresponding conic in the image, determine the pose of the world plane. The solution is in two stages:

(1) determine the orientation $R_{c e}$ of the plane,

(2) determine the orthogonal distance $d_{\perp}$ of the plane from the camera origin.

The method exploits the property, unique to a conic that is an ellipse, that the back-projected curve must have the following representation: $C_{0} X_{e}^{2}+A_{0} Y_{e}^{2}=1\left(A_{0}>0\right.$ and $\left.C_{0}>0\right)$ given in a canonical reference frame (principal axes reference frame $\left.R_{e}\right)$.

\section{(1) Plane orientation}

The conic $E$ in the image is given by (7). We assume that the origin is at the principal point, and the distances are measured in units of focal length. Then, the image curve defines a cone $a y^{2}+b x y+c y^{2}+d y z+e x z+f z^{2}=0$ in $3 \mathrm{D}$. The matrix in the quadratic form representation of this cone is $\mathbf{x}^{t} E \mathbf{x}=0$ (where $\mathbf{x}=(x, y, z)^{t}$ ) and may be diagonalized in the standard manner by a $3 \mathrm{D}$ rotation $R_{1}$ of the coordinate system to eigenvector frame. We have $E^{\prime}=R_{1}^{t} E R_{1}$ and $\mathbf{x}^{\prime}=R_{1}^{t} \mathbf{x}$, where $R_{1}=\left(\mathbf{e}_{1}, \mathbf{e}_{2}, \mathbf{e}_{3}\right)$ is the matrix of orthonormal eigenvectors and

$$
E^{\prime}=\left(\begin{array}{ccc}
\lambda_{1} & 0 & 0 \\
0 & \lambda_{2} & 0 \\
0 & 0 & \lambda_{3}
\end{array}\right)
$$

where $\left\{\lambda_{1}, \lambda_{2}, \lambda_{3}\right\}$ are the eigenvalues in ascending order ( $\lambda_{1}$ is then the only negative eigenvalue). Identification with coefficients $A_{0}$ and $C_{0}$ is achieved by a second rotation about the $y^{\prime}$ axis by an angle $\theta$ such that $\cos \theta=$ $\pm \sqrt{\left(\lambda_{3}-\lambda_{2}\left(C_{0} / A_{0}\right)\right) /\left(\lambda_{3}-\lambda_{1}\right)}$, which sets both coefficients to $\lambda_{2}$. There is therefore a four-fold ambiguity in the orientation $(\theta,-\theta, \theta+\pi, \pi-\theta)$. We have $E^{\prime \prime}=R_{2}^{t} E^{\prime} R_{2}$ and $\mathbf{x}^{\prime \prime}=R_{2}^{t} \mathbf{x}^{\prime}$ where

$$
R_{2}=\left(\begin{array}{ccc}
\cos \theta & 0 & \sin \theta \\
0 & 1 & 0 \\
-\sin \theta & 0 & \cos \theta
\end{array}\right)
$$

The composite rotation from image plane to the plane that intersects the cone in an ellipse is thus $\mathbf{x}^{\prime \prime}=R_{c e}^{t} \mathbf{x}$, where $R_{c e}=R_{1} R_{2}$ and, consequently, the normal to the plane in the camera coordinate system is $\mathbf{n}=R_{c e}(0,0,-1)^{t}$ (the -1 accounts for the right-handed coordinate system).

\section{(2) Plane distance}

In the $\mathbf{x}^{\prime \prime}=\left(x^{\prime \prime}, y^{\prime \prime}, z^{\prime \prime}\right)^{t}$ coordinate system, the ellipse has the equation

$$
C_{0}\left(x^{\prime \prime}-\alpha\right)^{2}+A_{0} y^{\prime \prime 2}=-\frac{A_{0}^{2} \lambda_{1} \lambda_{3}}{C_{0} \lambda_{2}^{2}} z^{\prime \prime 2}=1,
$$

with

$$
\begin{aligned}
& \alpha^{2}=\frac{\left(\lambda_{2}\left(C_{0} / A_{0}\right)-\lambda_{1}\right)\left(\lambda_{3}-\lambda_{2}\left(C_{0} / A_{0}\right)\right)}{\left(\lambda_{2}\left(C_{0} / A_{0}\right)\right)^{2}}, \\
& z^{\prime \prime}=d_{\perp}=\sqrt{-\frac{\left(\lambda_{2}\left(C_{0} / A_{0}\right)\right)^{2}}{C_{0} \lambda_{1} \lambda_{3}}}
\end{aligned}
$$

is the perpendicular distance of the plane from the origin. Finally, the ellipse center is at the position $\mathbf{t}_{c e}=R_{c e}\left(\alpha, 0, d_{\perp}\right)^{t}$ in the camera frame. To conclude this section, it is clear that values of $C_{0}$ and $A_{0}$ can be recovered if the orientation $R_{c e}$ of the plane and the orthogonal distance $d_{\perp}$ are provided elsewhere, by a camera calibration procedure for instance.

\section{RESULTS ANALYSIS}

Experimental investigations were performed on a winding plant composed of a winder, a traction motor and an unwinder. Figure 1 shows some details of our small web winding system. For experiments, velocity is typically about $100 \mathrm{~m} / \mathrm{min}$ which is a typical value in the web transport industry. An 8-bit digital CCD camera is placed in front of the winder. The image acquisition system consists of a DALSA CA-D8 $(512 \times 512)$ grey-scale digital camera $(80 \mathrm{i} / \mathrm{s})$ connected to a PC-DIG frame grabber by Imaging Technology. Video data transfer through PCI bus master to host memory is complete in less than 4 milliseconds. All image acquisitions and processings were done on a set of three Bi-Pentium III computers (connected through an ethernet D-Link gigabit 


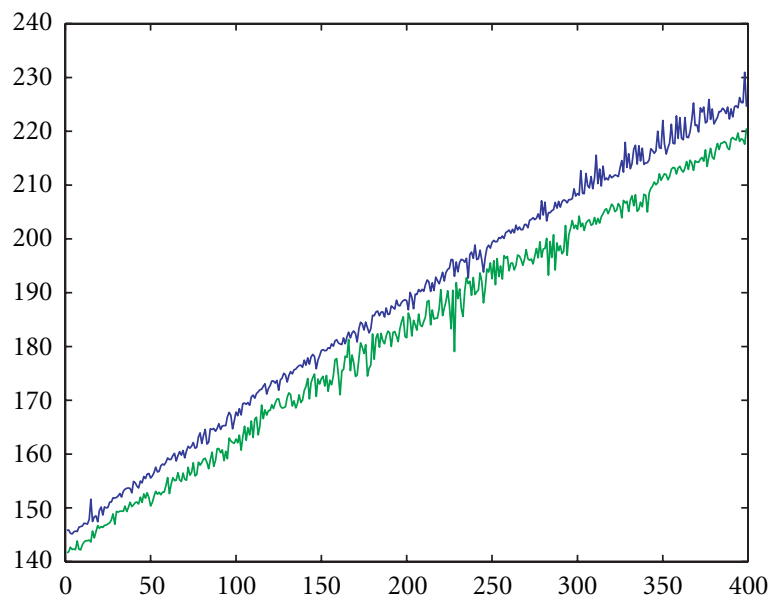

(a)

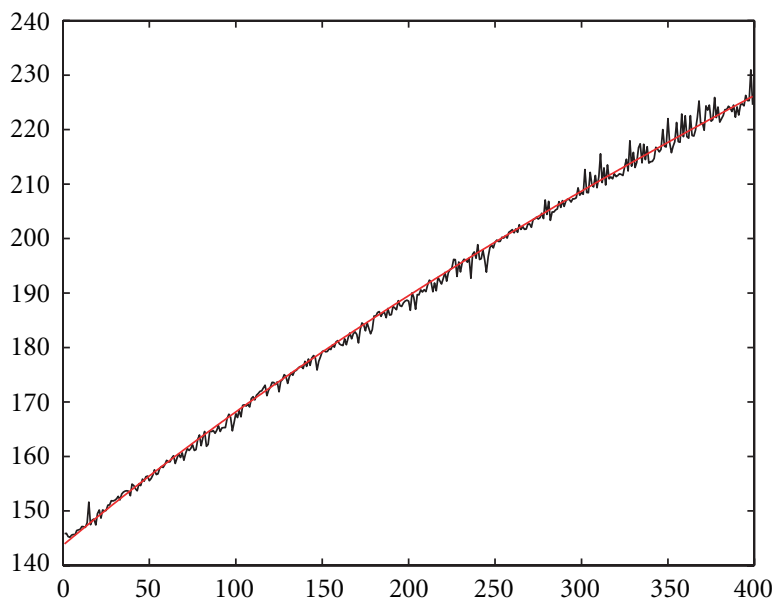

(b)

Figure 15: (a) Minor/major semiaxes of the roll along the image sequence. (b) Experimental results of the mean roll radius and its model (in pixels).

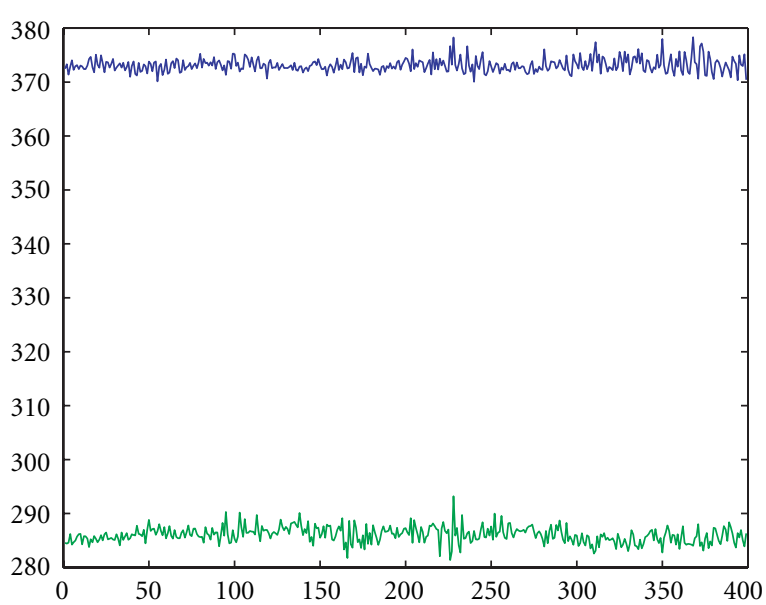

Figure 16: Estimations of the roll center coordinates (in pixels) along the image sequence.

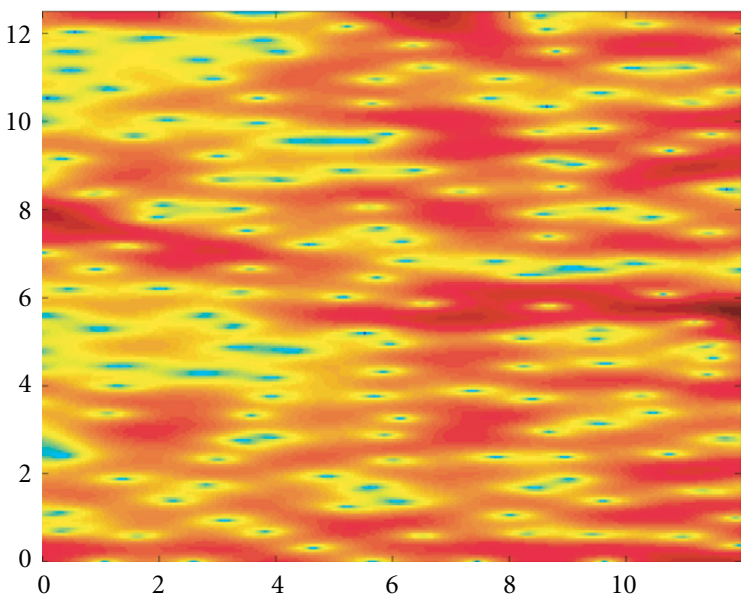

(a)

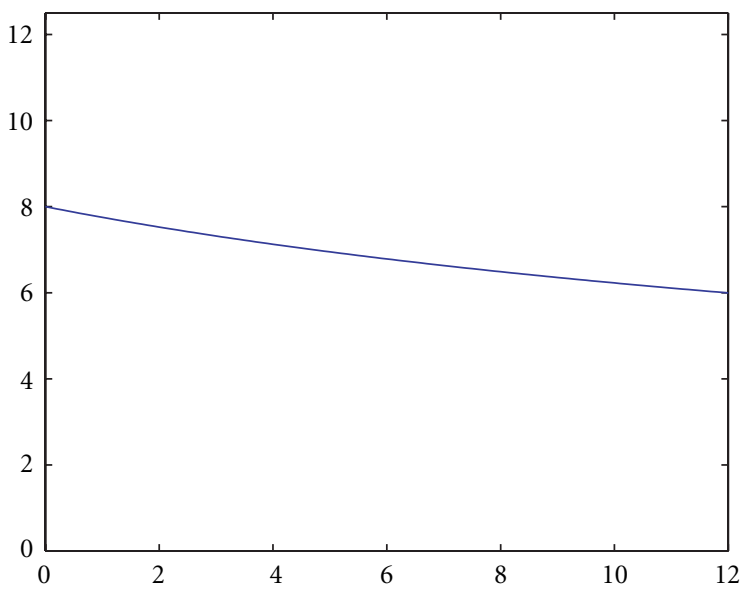

(b)

Figure 17: (a) Spectrogram of the sine-sliding behavior (from 8 to $6 \mathrm{~Hz}$ ) of the motion of the roll center. (b) Temporal model of angular velocity $\Omega(t)$ ( $a=0.068$, abscissa is unit time in second).

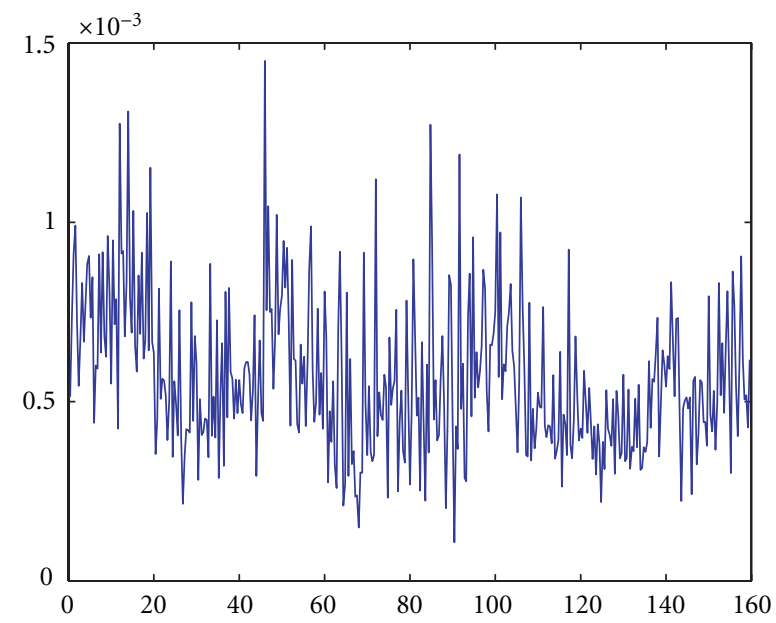

FIGURE 18: Mean square error of ellipse fitting (in pixels) for outer layer of the roll $\left(\times 10^{-3}\right)$. 

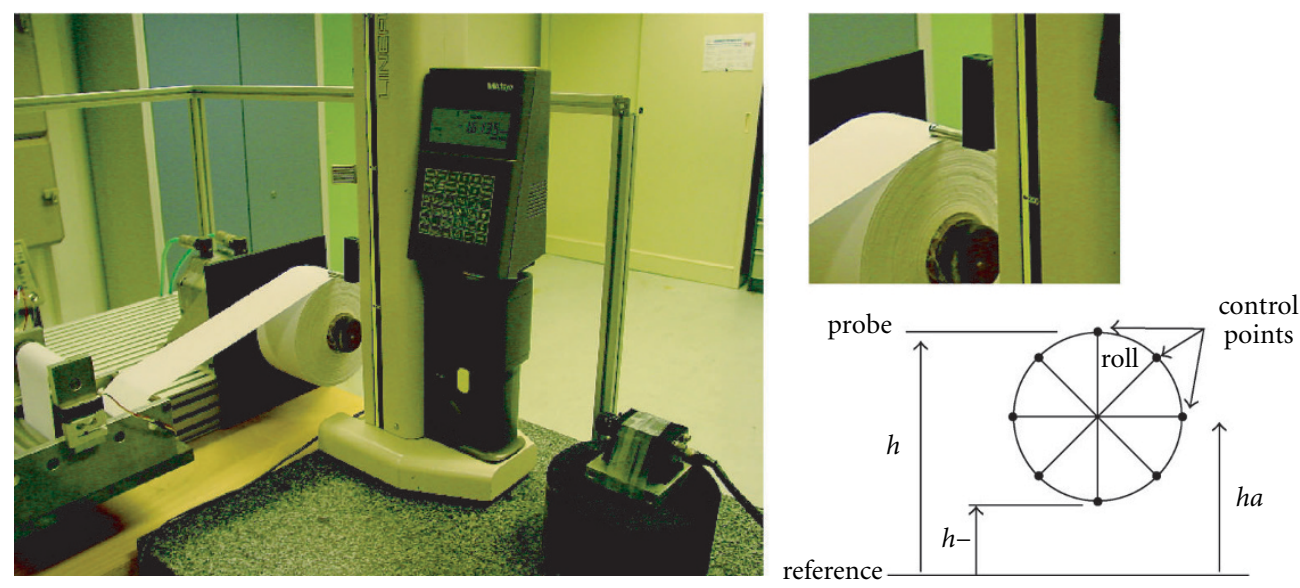

FIGURE 19: The linear height measuring machine and locations of control points along the roll.

network) and developped under the $\mathrm{C}++$ /MPI programming language. Today, the whole image processing is achieved at a video rate of about $25 \mathrm{i} / \mathrm{s}$. Figure 15 shows estimations of the minor and major semiaxes of the elliptic roll. As shown in Figure 15b, a mean value of the roll is computed for every image acquisition, compared with a model accounting for the radius increase and described below. This confirms the accuracy of the roll radius estimation in the image. The metric value of the roll radius can be found by means of a camera calibration [23] and the pose algorithm previously described. The computed mean square error of data points location from the estimated ellipse in Figure 18 is always less than $\sqrt{2.10^{-3}} \approx 1 / 20$ pixel all along the sequence, so this confirms our choice of an elliptical shape for the rolling winder profile.

In this paragraph, we modelize the increase of roll radius all along the sequence. It is of prime importance for an industrial process control to predict its value. Denote by $V$ and $\Omega$ the linear and angular winding velocity, respectively, by $r$ the roll radius (the rolling winder is assumed to be circular in this paragraph), and by $e$ the thickness of a web layer. Considering that a small increase of the roll radius is proportional to a small increase of the angle, then

$$
\begin{gathered}
r(t)=r(0)+\frac{e}{2 \pi} \int_{0}^{t} \Omega(t) d t \\
V=r(t) \Omega(t)
\end{gathered}
$$

Assuming that the sliding effect is of low significance along the web, then the winding velocity is identical to the unwinding velocity. The consequence of such hypothesis is that $d V / d t=0$ which leads to the simple differential equation

$$
r \frac{d^{2} r}{d t^{2}}+\left(\frac{d r}{d t}\right)^{2}=\frac{d}{d t}\left(r \frac{d r}{d t}\right)=0
$$

The solution for the radius is given by

$$
r(t)=r(0) \sqrt{1+a t}, \quad \text { with } a=\frac{e \Omega(0)}{\pi r(0)}=\frac{e V}{\pi r^{2}(0)} .
$$

From (19), the angular velocity can be also derived, $\Omega(t)=$ $\Omega(0) / \sqrt{(1+a t)}$. In spite of the fact that previous model has been built on pure kinematic considerations and with a rigid web, the course of the computed roll radius is very close to its estimates all along the sequence as shown in Figure 15b. Noise characteristics have been estimated to a value of 1.19 pixels for the standard deviation and 0.2 pixel for the mean.

Estimation of ellipse center coordinates are shown in Figure 16. Variations (typically of about 5 pixels) are due to the roll eccentricity. Frequency analysis was performed on the $x$-coordinate of the roll eccentricity. It relates a displacement of the main frequency mode and the angular velocity (see Figure 17). Although eccentricity is detected, noise is significant. A more accurate eccentricity detection could be achieved with more images per round (here, we typically have up to five images per round at $100 \mathrm{~m} / \mathrm{min}$ ).

In an attempt to validate the proposed method, we compare previous results for the roll radius estimates and its variations with those achieved straight with a high-accuracy height measuring machine. A Mitutoyo Linear Height LH600 has been installed on a marble surface. This machine provides an elevation course of $600 \mathrm{~mm}$ and can be easily moved on the plate. It incorporates a reflective-type linear encoder which has a resolution down to $0.5 \mu \mathrm{m}$. A $10 \mathrm{~mm}$ diameter ball probe is used for measuring the height $h$ with respect to the surface plate (the reference). We also defined eight control points per round by writing eight marks on the back side of the roll (see Figure 19). Since an accurate measure of the height $h_{a}$ of roll axis is performed, the difference $h-h_{a}$ provides a measurement value for the roll radius at each control point. We manually turned the roll of one eighth of a round to reach the next control point (exactly nine control points were used per round, the last one was identical to the first one up to a round). Since unwinding entirely the web in such a way is unpractical, we skip five rounds before beginning a new series of measurements. An image is grabbed by the camera for each series.

Figure 20a shows the results of 20 series of measurements. From this, the web thickness $e$ can be recovered $(e=280 \mu \mathrm{m})$ as $5 e$ is equal to the distance between each 


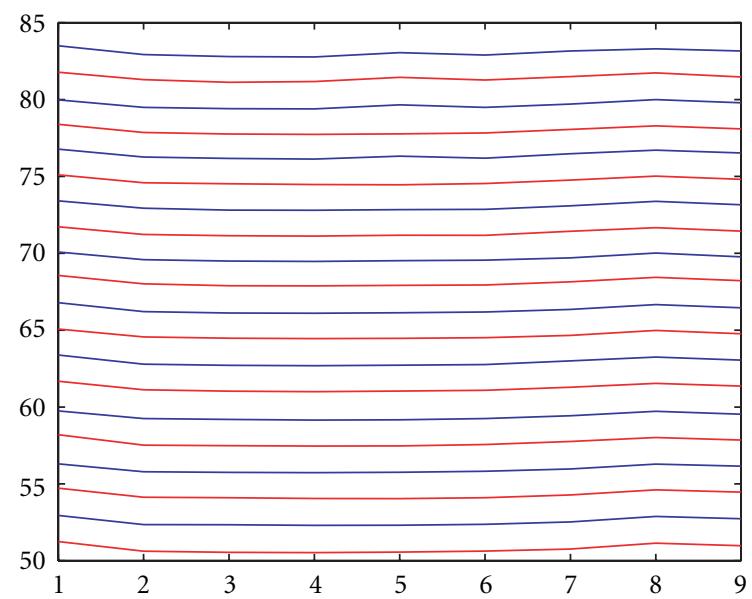

(a)

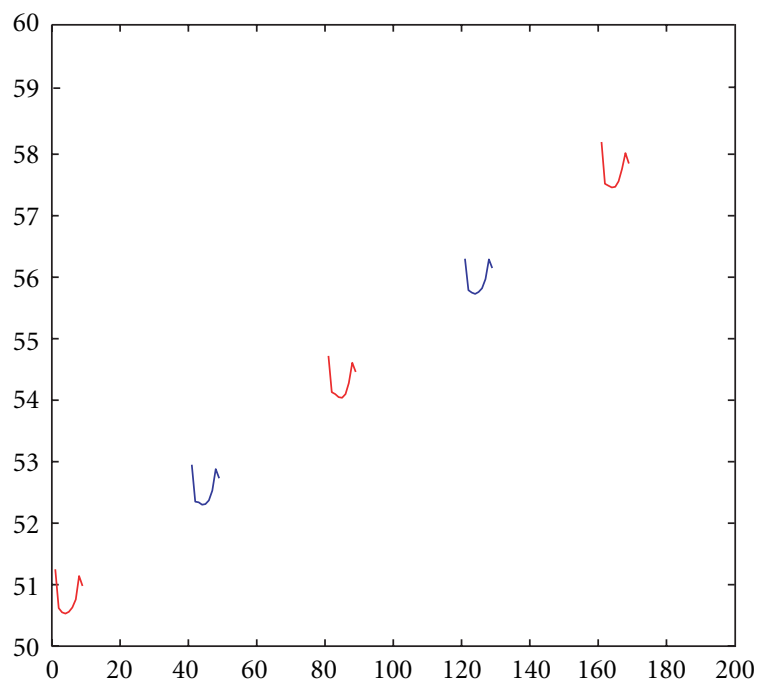

(b)

Figure 20: (a) 180 measurements of the roll radius (in $\mathrm{mm}$ ) with the LH600, (b) details of five series (45 measures) well arranged in the sequence of measures.

curve. Furthermore, it is clear that a significant noncircularity appears (see Figure 20b). To derive it, positions of control points all around the roll have been computed for each series in order to fit an ellipse.

Since an ellipse object (the roll) and its projection are both available, we can recover the orientation (only 2 degrees of freedom since the roll is quite circular) and the position of a reference object frame with respect to the camera frame provided that the camera calibration was previously done (the focal length value has been estimated in [23], and $f=11.59 \mathrm{~mm}$ for the standard $12 \mathrm{~mm}$ optical lens used). In fact, since the calibration plane (coplanar patterns) has been placed just in front of the roll and since all calibration procedures using geometrical features simultaneously estimate intrinsic and extrinsic parameters (parameters of the Euclidean transformation), these values may be used to backproject the ellipse image to the 3D space. Results (in $\mathrm{mm}$ )

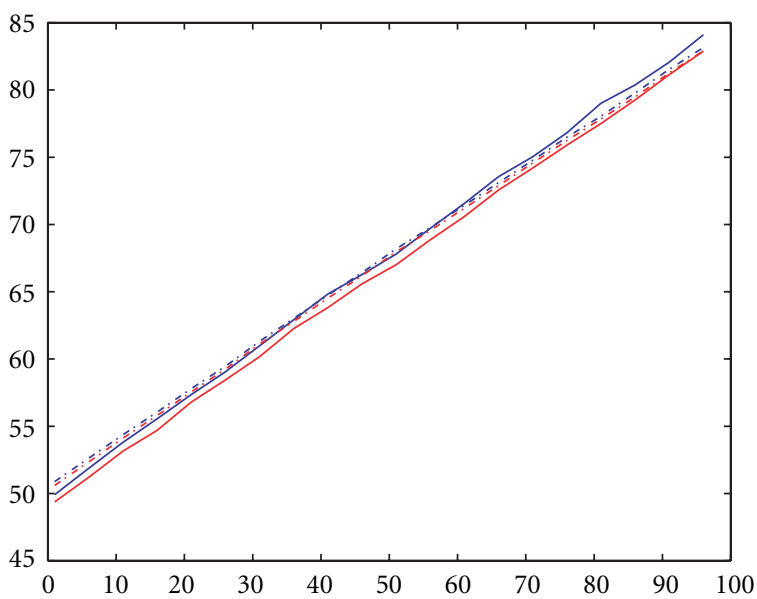

(a)

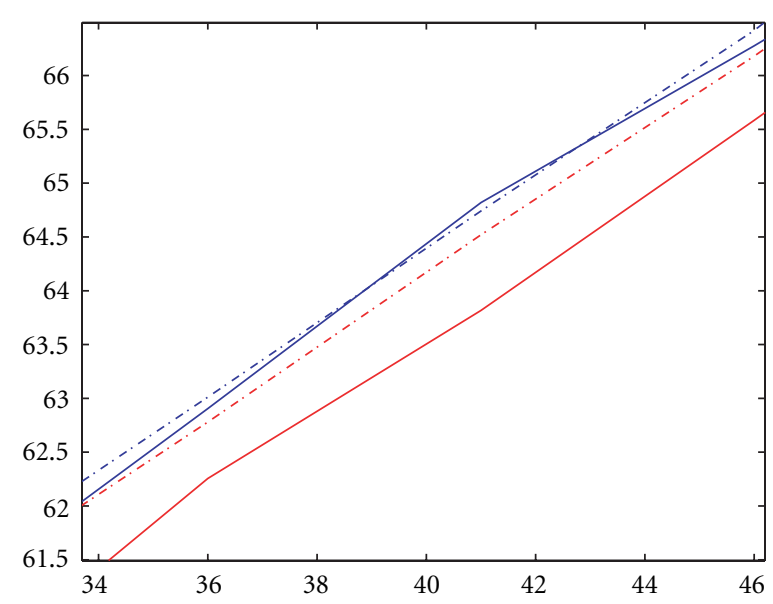

(b)

FIgURE 21: Comparison between minor/major semiaxes measurements (in $\mathrm{mm}$ ) on the static roll (dashed) and the back-projection to $3 \mathrm{D}$ space of minor/major semiaxes estimated from ellipse fitting (solid).

are shown in Figure 21 for the minor and major semiaxes (defined as $\sqrt{C_{0}}$ and $\sqrt{A_{0}}$ in Section 4) of the set of twenty ellipses. We observe in this figure that solid lines (estimations with the vision system) are closed to dashed lines (mechanical measurements) with an absolute accuracy of about $0.4 \mathrm{~mm}$ for the minor semiaxis and $0.1 \mathrm{~mm}$ for the major semiaxis (the mean value of the roll radius has an accuracy better than $0.3 \mathrm{~mm}(|\Delta r / r| \approx 0.5 \%))$.

\section{CONCLUSION}

This report presents the online detection and estimation of a roll radius and its variations (noncircularity and eccentricity) by the use of an imaging sensor applied to a web winding system. We describe a method for features extraction and classification based on wavelets transform which is well adapted for computer vision applications. In a real-time environment, the smoothing filter we propose to implement 
for the orientation function of edge contours is highly efficient in comparison with the Gaussian filter. It trades off maximum elimination of noise with preservation of the high frequency information inside the gradient phase signal.

Another contribution of this work is the improvement of the Pilu's technique for ellipse fitting. With a prenormalization of data and the use of Cholesky decomposition, an adequate criterion is proposed for analytically segregate ellipse from other conics. It is of most importance to take care of the fact that a common characteristic of methods based on the algebraic distance (the only methods providing analytical solutions for ellipse fitting with no approximation) is to be biased towards low-eccentricity for data covering a short arc of ellipse. That is why we focus on extracting data well-scattered along outer contours of the roll. We evaluate the validity of the proposed method by comparing the results obtained from the vision system to those measured with an accurate mechanical measuring machine. The roll radius has been well estimated with an accuracy (in 3D space) better than $0.3 \mathrm{~mm}$, since noncircularity and eccentricity of the roll have been taken into account all through this work.

\section{ACKNOWLEDGMENTS}

The authors wish to thank the French Ministry of Research for financial support by means of the ERT-Project "Highspeed handling and winding of flexible webs."

\section{REFERENCES}

[1] K. N. Reid and K.-C. Lin, "Control of longitudinal tension in multi-span web transport systems during start-up," in Proc. 2nd International Conference on Web Handling, pp. 77-95, Oklahoma city, Okla, USA, 1993.

[2] H. Koç, D. Knittel, M. De Mathelin, and G. Abba, "Modeling and robust control of winding for elastic webs," IEEE Transactions on Control System Technology, vol. 10, no. 2, 2002.

[3] W. Wolfermann, "Compensation of disturbances in the web force caused by a non-circular running winder," in Proc. International Conference on Web Handling, Oklahoma city, Okla, USA, 1999.

[4] S. Straub and D. Schröder, "An example of an application of neural networks in rolling mills: compensation of the noncircularity of winders," in IFAC Workshop on Motion Control, pp. 583-590, Munich, Germany, October 1995.

[5] B. Gueldenberg and Welp E. G., "Quantitative analysis of nip-induced tension by use of digital image processing," in Proc. International Conference on Web Handling, Oklahoma city, Okla, USA, June 1999.

[6] C. Doignon and D. Knittel, "Non-circularity detection of a rolling winder by artificial vision," in Proc. 5th IEEE International Conference on Quality Control by Artificial Vision, Le Creusot, France, May 2001.

[7] C. Daul, Construction et utilisation de listes de primitives en vue d'une analyse dimensionnelle de pièces à géométrie simpleApplication à la vision par ordinateur, Ph.D. thesis, Louis Pasteur University, Strasbourg, France, January 1994.

[8] D. P. Huttenlocher and S. Ullman, "Recognizing solid objects by alignment with an image," International Journal of Computer Vision, vol. 5, no. 2, pp. 195-212, 1990.

[9] D. M. Wuescher and K. L. Boyer, "Robust contour decomposition using a constant curvature criterion," IEEE Trans. on
Pattern Analysis and Machine Intelligence, vol. 13, no. 1, pp. 41-51, 1991.

[10] A. Pikaz and I. Dinstein, "Using simple decomposition for smoothing and feature point detection of noisy digital curves," IEEE Trans. on Pattern Analysis and Machine Intelligence, vol. 16, no. 8, pp. 808-813, 1994.

[11] I. Weiss, "High-order differentiation filters that work," IEEE Trans. on Pattern Analysis and Machine Intelligence, vol. 16, no. 7, pp. 734-739, 1994.

[12] I. Weiss, "Noise-resistant invariants of curves," IEEE Trans. on Pattern Analysis and Machine Intelligence, vol. 15, no. 9, pp. 943-948, 1993.

[13] V. Govindu and C. Shekhar, "Alignment using distributions of local geometric properties," IEEE Trans. on Pattern Analysis and Machine Intelligence, vol. 21, no. 10, pp. 1031-1043, 1999.

[14] J. Fayolle, L. Riou, and C. Ducottet, "Robustness of a multiscale scheme of feature points detection," Pattern Recognition, vol. 33, no. 9, pp. 1437-1453, 2000.

[15] N. Werghi and C. Doignon, "Contour decomposition with wavelet transform and parametrisation of elliptic curves with an unbiased extended Kalman filter," in Proc. 2nd Asian Conference on Computer Vision, vol. 3, pp. 186-190, Singapore, December 1995.

[16] S. G. Mallat, "A theory for multiresolution signal decomposition," IEEE Trans. on Pattern Analysis and Machine Intelligence, vol. 11, no. 7, pp. 674-693, 1989.

[17] Y. Meyer, Les Ondelettes: Algorithmes et Applications, Armand Colin, Paris, 1992.

[18] C. C. Hsu and J. Huang, "Partitionned Hough transform for ellipsoid detection," Pattern Recognition, vol. 23, no. 3, pp. 275-282, 1990.

[19] P. L. Rosin and G. A. W. West, "Segmenting curves into elliptic arcs and straight lines," in Proc. 3rd International Conf. on Computer Vision, pp. 75-78, Osaka, Japan, November 1990.

[20] J. Porril, "Fitting ellipses and predicting confidence envelopes using a bias corrected Kalman filter," Image and Vision Computing, vol. 8, no. 1, pp. 37-41, 1990.

[21] M. Pilu, A. W. Fitzgibbon, and R. B. Fisher, "Ellipse-specific direct least-square fitting," in Proc. IEEE International Conference on Image Processing, pp. 599-602, Lausanne, Switzerland, September 1996.

[22] M. Dhome, M. Richetin, J. T. Lapresté, and G. Rives, "Spatial localization of modeled objects of revolution in monocular perspective vision," in Proc. 1st European Conference on Computer Vision, pp. 475-484, Antibes, France, April 1990.

[23] C. Doignon and G. Abba, "A practical multi-plane method for a low-cost calibration technique," in Proc. 5th European Control Conference, Karlsruhe, Germany, September 1999.

Christophe Doignon received the B.S. degree in Physics in 1987 and the Engineering diploma in 1989 both from the École Nationale Supérieure de Physique de Strasbourg, France. He received the Ph.D. degree in computer vision and robotics from Louis Pasteur University, Strasbourg, France in 1994. In 1995 and 1996, he worked with the department of Electronics and Computer Science at University of Padua, Italy, for the

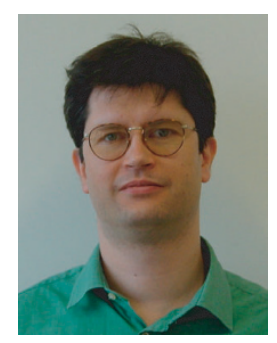
European Community under HCM program "Model Based Analysis of Video Information." Since 1996, he is Associate Professor in computer engineering at Louis Pasteur University, Strasbourg, France. His major research interests include computer vision, signal and image processing, visual servoing and robotics. 
Dominique Knittel received the Engineering diploma from the École Nationale Supérieure de Physique de Strasbourg, in 1987 and the Ph.D. degree from the University of Strasbourg in 1990, both in automatic control. Since 1992, he has been at University of Strasbourg, where he is currently an Associate Professor. His research interests include modeling, identification, robust control, control of large scale sys-

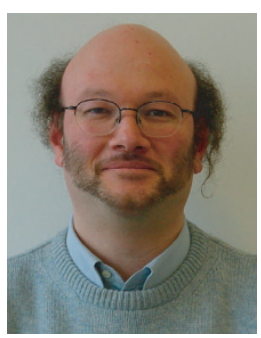

tems, mainly for winding systems. Since 1999 he has been the Team Manager of the ERT Project "High speed handling and winding of flexible webs." 